\title{
Galactosyltransferases from Arabidopsis thaliana in the biosynthesis of type II arabinogalactan: molecular interaction enhances enzyme activity
}

\author{
Adiphol Dilokpimol ${ }^{1,4 \dagger}$, Christian Peter Poulsen ${ }^{1 \dagger}$, György Vereb², Satoshi Kaneko ${ }^{3}$, Alexander Schulz
} and Naomi Geshi ${ }^{1^{*}}$

\begin{abstract}
Background: Arabinogalactan proteins are abundant proteoglycans present on cell surfaces of plants and involved in many cellular processes, including somatic embryogenesis, cell-cell communication and cell elongation. Arabinogalactan proteins consist mainly of glycan, which is synthesized by post-translational modification of proteins in the secretory pathway. Importance of the variations in the glycan moiety of arabinogalactan proteins for their functions has been implicated, but its biosynthetic process is poorly understood.

Results: We have identified a novel enzyme in the biosynthesis of the glycan moiety of arabinogalactan proteins. The At1g08280 (AtGALT29A) from Arabidopsis thaliana encodes a putative glycosyltransferase (GT), which belongs to the Carbohydrate Active Enzyme family GT29. AtGALT29A co-expresses with other arabinogalactan GTS, AtGALT31A and AtGLCAT14A. The recombinant AtGALT29A expressed in Nicotiana benthamiana demonstrated a galactosyltransferase activity, transferring galactose from UDP-galactose to a mixture of various oligosaccharides derived from arabinogalactan proteins. The galactose-incorporated products were analyzed using structure-specific hydrolases indicating that the recombinant AtGALT29A possesses $\beta$-1,6-galactosyltransferase activity, elongating $\beta-1,6$-galactan side chains and forming 6-Gal branches on the $\beta-1,3-$ galactan main chain of arabinogalactan proteins. The fluorescence tagged AtGALT29A expressed in N. benthamiana was localized to Golgi stacks where it interacted with AtGALT31A as indicated by Förster resonance energy transfer. Biochemically, the enzyme complex containing AtGALT31A and AtGALT29A could be co-immunoprecipitated and the isolated protein complex exhibited increased level of $\beta$-1,6-galactosyltransferase activities compared to AtGALT29A alone.
\end{abstract}

Conclusions: AtGALT29A is a $\beta-1,6$-galactosyltransferase and can interact with AtGALT31A. The complex can work cooperatively to enhance the activities of adding galactose residues 6 -linked to $\beta-1,6-$ galactan and to $\beta-1,3$-galactan. The results provide new knowledge of the glycosylation process of arabinogalactan proteins and the functional significance of protein-protein interactions among O-glycosylation enzymes.

Keywords: Arabidopsis thaliana, Arabinogalactan protein, Galactosyltransferase, Protein O-glycosylation, Golgi apparatus, Protein-protein interaction, FRET, Plant cell wall

\footnotetext{
* Correspondence: nge@plen.ku.dk

${ }^{\dagger}$ Equal contributors

'Department of Plant and Environmental Sciences, Thorvaldsensvej 40,

1871 Frederiksberg, C, Denmark

Full list of author information is available at the end of the article
}

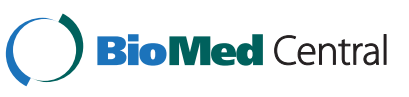

(c) 2014 Dilokpimol et al.; licensee BioMed Central Ltd. This is an Open Access article distributed under the terms of the Creative Commons Attribution License (http://creativecommons.org/licenses/by/2.0), which permits unrestricted use, distribution, and reproduction in any medium, provided the original work is properly credited. The Creative Commons Public Domain Dedication waiver (http://creativecommons.org/publicdomain/zero/1.0/) applies to the data made available in this article, unless otherwise stated. 


\section{Background}

Arabinogalactan proteins (AGPs) are an abundant class of proteoglycans in plant cell walls and are implicated in the control of cell proliferation and morphogenesis [1]. Numerous studies using monoclonal antibodies have demonstrated the developmentally regulated appearance of specific glycan epitopes correlated with changes in anatomy (for examples, [2-11]). Hence subtle differences in the glycan structure of AGPs may function as markers used in coordinating developmental processes in plants. However, defined structural features of the active AGP glycans have not been identified and their molecular specificity is unknown.

The glycans of AGPs originate by post-translational modification of protein backbones catalyzed by glycosyltransferases (GTs) in the secretory pathway. The glycan structure of AGPs is heterogeneous, but commonly composed of a $\beta$-1,3-linked galactan backbone with substitution of the side chains at $O 6$ positions (type II AG). The side chains are typically $\beta$-1,6-galactans, usually modified with arabinose (Ara) and less frequently with other sugars such as rhamnose (Rha), fucose (Fuc), and (4-O-methyl) glucuronic acid (GlcA) [12-14]. It is anticipated that more than 10 functionally distinct GTs are required to build the AGP glycans, and so far fucosyltransferases (AtFUT4, AtFUT6) [15], galactosyltransferases (AtGALT2 [16] and AtGALT31A [17]), and a glucuronosyltransferase (AtGLCAT14A) [18] have been characterized.

We have characterized an Arabidopsis GT encoded by At1g08280, which is co-expressed with AtGALT31A [17] and AtGLCAT14A [18]. This protein belongs to GT29 family in the Carbohydrate Active Enzyme database (CAZy, http://www.cazy.org) [19]. The GT29 family contains large numbers of eukaryotic and viral sialyltransferases acting on glycoproteins and/or glycolipids [20]. Several plant sequences have been placed in this family, and two of the rice sequences expressed in COS-7 cells showed sialyltransferase activity [21]. Arabidopsis has three proteins in this family (encoded by At1g08280, At1g08660 and At3g48820). Two of them (At1g08280 and At3g48820) expressed in COS-7 cells and in Nicotiana benthamiana, respectively, lacked sialyltransferase activity [21,22].

In this paper, we provide evidence for (i) $\beta-1,6$ galactosyltransferase (GalT) activity, encoded by At1g08280 in the biosynthesis of type II AG structure, (ii) its interaction with AtGALT31A, and (iii) an increase of $\beta-1,6$ GalT activity by the protein complex in an in vitro assay.

\section{Results}

At1g08280 is co-expressed with other type II arabinogalactan glycosyltransferases

The protein encoded by At1g08280 is predicted to have a single transmembrane domain at Val5-Ile27, a typical type II membrane topology commonly found in GTs. The transcript levels are generally low in Arabidopsis throughout development, but higher during seed maturation and root development, and the gene is co-expressed with AtGALT31A [17] and AtGLCAT14A [18], which were recently identified as possessing galactosyltransferase and glucuronosyltransferase activity, respectively, involved in the glycosylation of type II AGs (GeneCAT, http://genecat.mpg.de) [23] (Additional file 1: Figure S1). Therefore, we presumed that the activity encoded by At1g08280 may be involved in the glycosylation pathway of type II AGs, and investigated this hypothesis by biochemical assays using the protein expressed heterologously.

\section{Recombinant protein encoded by At1g08280 showed galactosyltransferase activity towards type II arabinogalactan acceptors}

For biochemical characterization, the full-length At1g08280 construct harboring $\mathrm{N}$-terminal HA tag was expressed in $N$. benthamiana and affinity purified using monoclonal anti-HA-antibody conjugated to agarose. The HA-At1g08280 collected on the bead slurry was used as the enzyme source for identification of donor substrate. We identified the donor substrate by testing 7 different NDP- $\left[{ }^{14} \mathrm{C}\right]$-sugars according to the methods $[17,18]$. We used microsomes prepared from $N$. benthamiana after expression of a synthetic peptide composed of a consensus sequence for AG glycosylation as acceptor for the assay $\left(\mathrm{GAGP}_{8}\right.$-GFP; [24]). This acceptor represents a mixture of various type II AG polysaccharides (for details of the structure, see [17]). When substrate mixtures were tested, we observed higher level of $\left[{ }^{14} \mathrm{C}\right]$-sugar incorporation from a mixture of UDP- $\left[{ }^{14} \mathrm{C}\right]$-GlcNAc, UDP- $\left[{ }^{14} \mathrm{C}\right]-$ GlcA and UDP- $\left[{ }^{14} \mathrm{C}\right]$-Gal (Mix II in Figure 1A) than from one containing UDP- $\left[{ }^{14} \mathrm{C}\right]-\mathrm{Xyl}$, UDP- $\left[{ }^{14} \mathrm{C}\right]-\mathrm{Glc}$, GDP- $\left[{ }^{14} \mathrm{C}\right]$-Man and GDP- $\left[{ }^{14} \mathrm{C}\right]$-Fuc (Mix I). When testing each substrate in the Mix II separately, we found UDP- $\left[{ }^{14} \mathrm{C}\right]-$ Gal works as a substrate (Figure $1 \mathrm{~B}$ ). The result indicates that the enzyme possesses a GalT activity, therefore, we named the enzyme AtGALT29A (Arabidopsis thaliana galactosyltransferase from family GT29).

\section{AtGALT29A Is localized to Golgi apparatus and interacts with AtGALT31A}

We determined the subcellular localization of AtGALT29A by transient expression of the C-terminal monomeric CFP (mCer3) fusion protein in N. benthamiana (Figure 2). The overlay of AtGALT29A-mCer3 with the co-expressed Golgi marker protein, $\mathrm{ST}_{\text {tmd }}$-YFP [25] indicated its localization to the Golgi apparatus.

Previously, AtGALT31A and AtGLCAT14A were also shown to be localized to the Golgi apparatus $[17,18]$. 

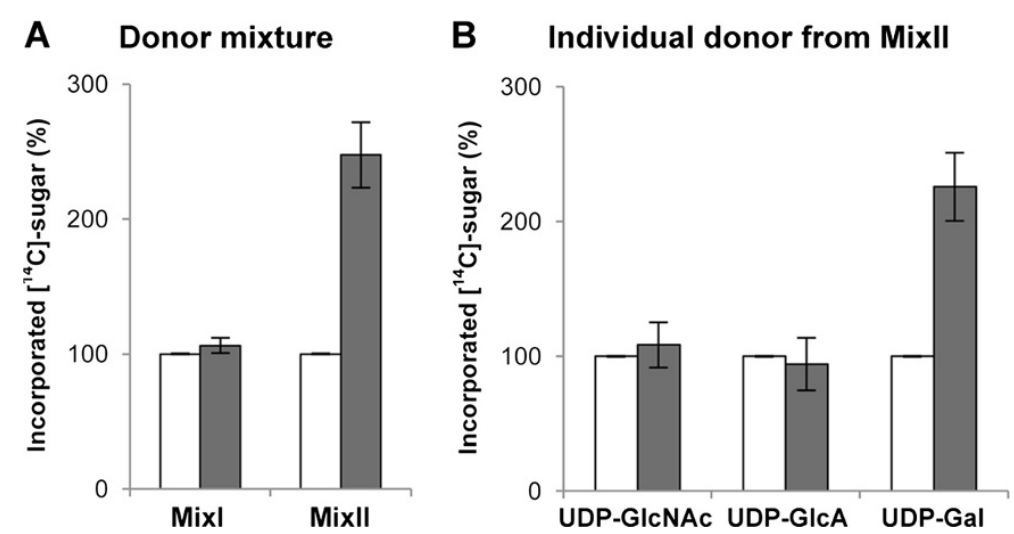

Figure 1 Identification of donor substrate for recombinant AtGALT29A. Affinity purified AtGALT29A (ש) or P19 ( $\square$ ) was incubated with A: NDP- $\left[{ }^{14} \mathrm{C}\right]$-sugars: UDP- $\left[{ }^{14} \mathrm{C}\right]-\mathrm{Xyl}$, UDP- $\left[{ }^{14} \mathrm{C}\right]-\mathrm{Gl}$, GDP- $\left[{ }^{14} \mathrm{C}\right]-$ Man and GDP- $\left[{ }^{14} \mathrm{C}\right]-$ Fuc (as Mixl), and UDP- $\left[{ }^{14} \mathrm{C}\right]-\mathrm{Gl} \mathrm{cNAC}$, UDP- $\left[{ }^{14} \mathrm{C}\right]-\mathrm{Gl} \mathrm{CA}$ and UDP- $\left[{ }^{14} \mathrm{C}\right]$-Gal (as Mixll); B: or individual NDP- $\left[{ }^{14} \mathrm{C}\right]$-sugars from Mixll using $G A G P_{8}$ as acceptor substrate. Error bars showed standard deviations from $n=4$. The result indicates that UDP- $\left[{ }^{14} \mathrm{C}\right]-$ Gal serves substrate for AtGALT29A.

AtGALT29A-YFP was co-localized with AtGALT31AmCer3 to a high degree (approximately $80 \%$, Figure $3 \mathrm{~A}-\mathrm{C}$ ), while AtGALT29A-mCer3 and AtGLCAT14A-YFP were only partially co-localized (approximately 52\%, Additional file 2: Figure S2A-C). Next, we tested protein-protein interaction within and between AtGALT29A and AtGALT31A using the FRET acceptor photobleaching technique for these proteins tagged with either mCer3 or YFP ectopically expressed in $N$. benthamiana [26,27]. FRET from mCer3 (donor) to YFP (acceptor) happens when the two fluorescent proteins are closer than $10 \mathrm{~nm}$, indicative of interaction between the tagged proteins. Bleaching of the acceptor YFP allows measuring absolute FRET efficiency in a self-controlled manner [26,27], so values above 0 definitely indicate molecular interaction between the tagged proteins. When the homodimeric combinations (AtGALT31A-mCer3 + AtGALT31A-YFP and AtGALT29A-mCer3 + AtGALT29A-YFP, respectively) were tested, FRET efficiencies of $19 \%$ and $34 \%$ were assessed, respectively (Figure 3D, 3F), indicating the formation of homodimers for both AtGALT31A and AtGALT29A. When AtGALT31A-mCer3 + AtGALT29A-YFP and AtGALT29AmCer3 + AtGALT31A-YFP were co-expressed, FRET efficiencies of $18 \%$ and $29 \%$ were detected, respectively, indicating the formation of heterodimers between AtGALT29A and AtGALT31A (Figure 3E, 3G). Therefore we observed positive interactions for all combinations tested (Figure 3), but differences in the values of FRET efficiencies are evident, when these are calculated on a pixel-by-pixel analysis. When AtGALT29A is the donor (mCer3 tagged, Figure $3 \mathrm{~F}$ and $3 \mathrm{G}$ ), FRET efficiencies are overall higher (34\% and 29\%) compared to the combinations when AtGALT31A is the donor (19\% and $18 \%$, Figure 3D and 3E). Thus, AtGALT31A-mCer3 is either less able to dimerize than AtGALT29A-mCer3 under the experimental conditions, or is in a conformation which is less efficient as a donor. Nevertheless, when we use the same donor (either AtGALT29A-mCer3 or AtGALT31A-mCer3), and compare FRET efficiencies for homo and heterodimerization, we obtain roughly the same FRET efficiency
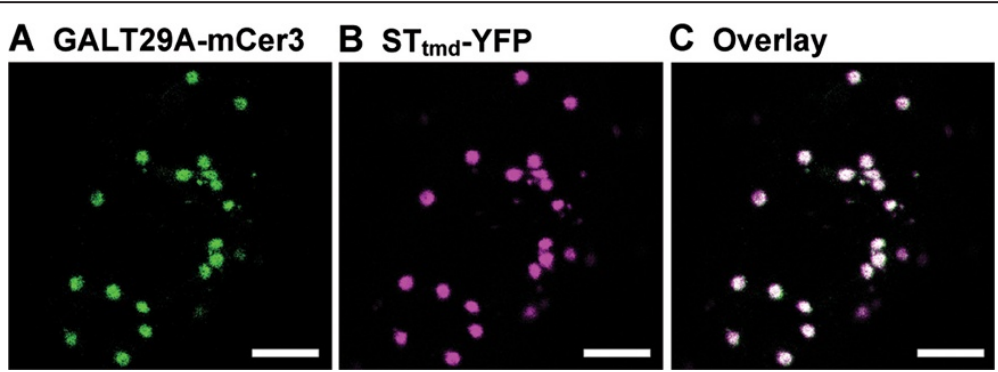

Figure 2 Subcellular localization of AtGALT29A-mCer3 in N. benthamiana leaves. A-B: Confocal images of AtGALT29A-mCer3, ST tmd-YFP (a Golgi marker) co-expressed transiently in $\mathbf{N}$. benthamiana leaves. C: The overlay image of (A) and (B). The result indicates co-localization of ATGALT29A-mCer3 and ST tmd $^{-Y F P}$ in the Golgi apparatus. Scale bar $=5 \mu \mathrm{m}$. 

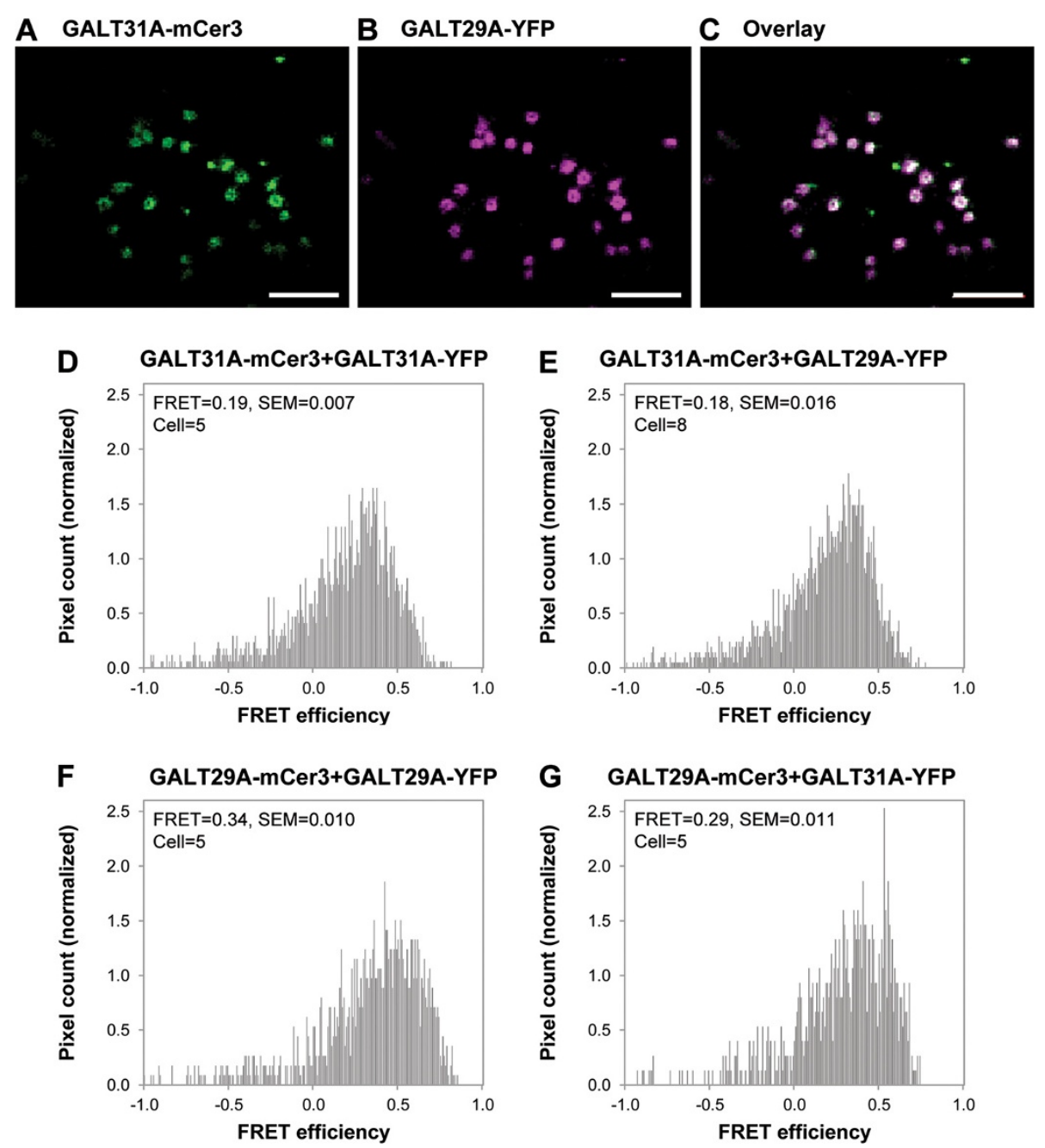

Figure 3 Localization and FRET analysis for AtGALT29A and AtGALT31A. A-B: Confocal images of AtGALT31A-mCer3 and AtGALT29A-YFP co-expressed in N. benthamiana leaves. C: The overlay image of (A) and (B). AtGALT31A-mCer3 and AtGALT29A-YFP are co-localized in high frequency. D-G: Distribution histogram for pixel by pixel analysis of FRET [26]. FRET efficiency is expressed as FRET=, for example, FRET =0.19 in (D) means that FRET efficiency is 19\%; SEM, standard error of means; cell = number of cells analyzed. Scale bar $=5 \mu \mathrm{m}$.

for homo and heterodimers. For AtGALT29A-mCer3/ AtGALT29A-YFP and AtGALT29A-mCer3/AtGALT31AYFP we obtain $34 \%$ and $29 \%$, (Figure $3 \mathrm{~F}$ and $3 \mathrm{G}$, respectively), indicating that the AtGALT29A-mCer3/ AtGALT31A-YFP heterodimer is preferred to the AtGALT29A homodimer, since in spite of the possibility of homodimer formation in the AtGALT29A-mCer3/ AtGALT31A-YFP system, which could decrease FRET by incorrect donor/acceptor pairing, we still have the same level of FRET efficiency as when we have only AtGALT29A. The same tendency is also observed when AtGALT31A-mCer is the donor (19\% and $18 \%$, Figure $3 \mathrm{D}$ and $3 \mathrm{E}$, respectively).

Overall, our results indicate the formation of homodimers for both AtGALT31A and AtGALT29A as well as that of heterodimers between them when these two GTs were expressed simultaneously. The indicated interactions are unlikely to be due to an overexpression artifact since AtGALT31A and AtGLCAT14A did not interact under the same experimental set up [18]. AtGALT29A also interacted with AtGLCAT14A when the two proteins were co-localized (13\% mean FRET efficiency, Additional file 2: Figure S2D). But, since AtGALT29A and AtGLCAT14A were only occasionally co-localized, occurrence of the interaction between these two proteins is considered to be of less importance than that between AtGALT29A and AtGALT31A.

AtGALT31A is co-purified with AtGALT29A as an enzyme complex and increases the level of galactose incorporation into the type II AG acceptors

Since FRET analysis indicated molecular interactions between AtGALT31A and AtGALT29A (Figure 3), we tried to purify the enzyme complex and investigated GalT 
activity when AtGALT29A is alone or in a complex with AtGALT31A. We expressed AtGALT31A as a C-terminal GFP fusion protein (AtGALT31A-GFP) and AtGALT29A as an N-terminally HA tagged protein (HA-AtGALT29A) in $N$. benthamiana, and immunoprecipitated the enzyme complex using an anti-GFP antibody (Figure 4A). When AtGALT31A-GFP was expressed alone, it was immunoprecipitated as a band of ca. $70 \mathrm{kDa}$ using Western blot analysis with the same antibody (Figure 4A, lane 2). The corresponding band was also detected in the immunoprecipitated material using anti-HA resin from the co-expression sample of both proteins (Figure 4A, lane 5). This indicates co-purification of AtGALT31A with AtGALT29A using a tag on AtGALT29A, thus the complex formation indicated by the FRET analysis was also confirmed by co-immunoprecipitation (Figure 3). The band around $50 \mathrm{kDa}$ detected in lanes $3-5$ is the heavy chain of the HA antibody used for immunoprecipitation, which was somehow detected by the secondary antibody in the Western blot analysis.

We attempted to evaluate the purity of the protein complex(es) by eluting the immobilized complex(es) from the anti-HA agarose slurry using low $\mathrm{pH}$ buffer as recommended by the manufacturer; however, the majority of the proteins were not eluted to the buffer in an amount detectable by Western blot analysis (data not shown). When the immunoprecipitated samples collected on anti-HA antibody-agarose were directly subjected to SDS-PAGE and analyzed by the Western blot, we could detect the recombinant proteins (Figure 4).

Using the immunoprecipitated enzyme complex, we investigated GalT activity in the biosynthesis of type II AG using UDP- $\left[{ }^{14} \mathrm{C}\right]-\mathrm{Gal}$ as donor-substrate and $\mathrm{SP}_{32-}$ GFP as acceptor, which is microsomes prepared from

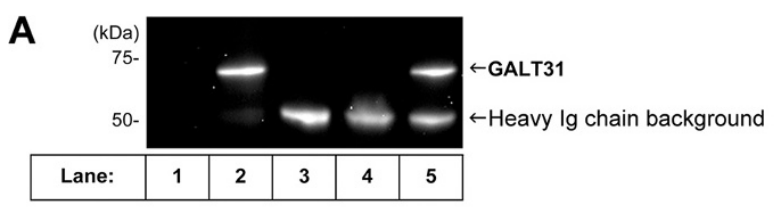

B
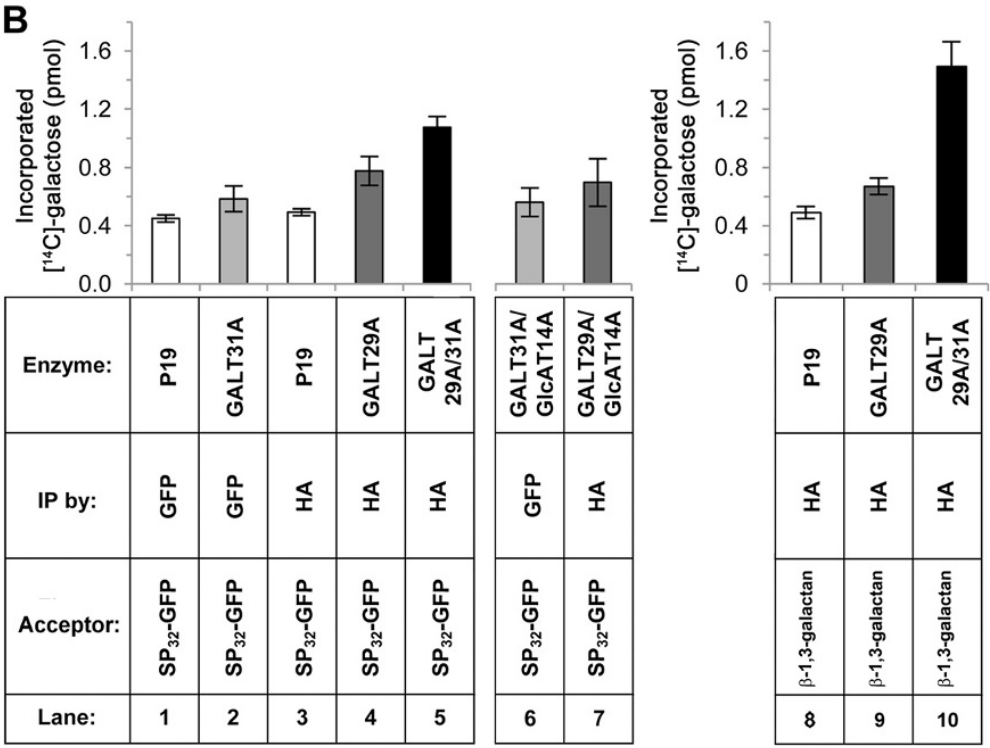

Figure 4 Galactosyltransferase activity using the purified AtGALT29A/AtGALT31A complex in vitro. Microsomes were prepared from N. benthamiana leaves after expression of P19 only, AtGALT31A-GFP, HA-AtGALT29A or co-expression of HA-AtGALT29A and AtGALT31A-GFP, and subjected to immunoprecipitation using anti-GFP- or anti-HA-antibody. The conditions are indicated in the table at the bottom of (B). The immunoprecipitated samples were analyzed by the Western blot (A) and by the enzyme activity (B). A: The Western blot of P19,

AtGALT31A-GFP, HA-AtGALT29A and AtGALT29A/AtGALT31A immunoprecipitated using GFP antibody. The result indicates co-purification of AtGALT31A-GFP (lane 5, indicated by the arrow at ca. $70 \mathrm{kDa}$ ) by immunoprecipitation of HA-AtGALT29A using anti-HA-antibody-agarose. The $50 \mathrm{kDa}$ band detected in the lanes 3-5 is the heavy chain of HA antibody used for the immunoprecipitation, which is recognized by the secondary antibody used in the Western blot. B: Galactosyltransferase activity towards SP ${ }_{32}$-GFP and $\beta-1,3-$ galactan acceptors. Affinity purified materials from the expression of P19 only, AtGALT31A-GFP, HA-AtGALT29A, or co-expression of HA-AtGALT29A and AtGALT31A-GFP using anti-GFP- or anti-HA-antibody were tested for enzyme activity using UDP. $-{ }^{14}[\mathrm{C}]-\mathrm{Gal}$ as substrate and SP 32 -GFP (lanes 1-5) or $\beta$-1,3-galactan (lanes 8-10) as acceptor, $(n=4)$. Control samples after co-expression of AtGALT31A-GFP or HA-AtGALT29A with HA-AtGLCAT14A (lane 6 and 7) were immunoprecipitated in the same way as for other samples and tested for the enzyme activity using UDP- ${ }^{14}[\mathrm{C}]-\mathrm{Gal}$ as substrate and $\mathrm{SP}_{32}-\mathrm{GFP}$ as acceptor (lanes 6-7), $(\mathrm{n}=3)$. These combinations are not suggested to form protein complexes based on the FRET analysis. Error bars showed standard deviations. 
$N$. benthamiana after expression of a consensus motifs for AG glycosylation, repetitive Ser-Pro [28]. This material contains various AG oligosaccharides similarly as detected in $\mathrm{GAGP}_{8}$ (see method). The protein complex containing AtGALT29A and AtGALT31A exhibited a higher level of $\left[{ }^{14} \mathrm{C}\right]-\mathrm{Gal}$ incorporation to the $\mathrm{SP}_{32}$ GFP acceptor compared to AtGALT29A alone (Figure 4B). While such an increase was not observed for the combination of AtGALT31A/AtGLCAT14A and AtGALT29A/ AtGLCAT14A (lane 6 and 7 in Figure 4B), indicating the increase of enzyme activity is specific by the combination between AtGALT29A and AtGALT31A.

Moreover, the enzyme complex showed higher levels of $\left[{ }^{14} \mathrm{C}\right]-\mathrm{Gal}$ incorporation also towards $\beta$-1,3-galactan acceptor by the enzyme complex compared to AtGALT29A alone (lane 8-10 in Figure 4B). The results indicate an increase of GalT activity towards both $\mathrm{SP}_{32}$-GFP and $\beta-1,3-$ galactan AG acceptors by the enzyme complex containing AtGALT31A and AtGALT29A when compared to a single enzyme.

The enzyme complex containing AtGALT31A and AtGALT29A exhibited increased $\beta-1,6-$ GalT activity adding Gal residues at 06 positions of $\beta-1,6-$ galactan and to $\beta-1$, 3-galactan

The $\mathrm{SP}_{32}$-GFP and $\beta$-1,3-galactan used in Figure 4 are composed of heterogeneous oligosaccharides: $\mathrm{SP}_{32}$-GFP prepared from microsomes consists of various components with different molecular size (ca. $40 \mathrm{kDa}, 75-100 \mathrm{kDa}$, larger than $150 \mathrm{kDa}$ ) and contains $\beta$-1,6-galactan side chains of a degree of polymerization (DP) from 1 to at least 8 , as well as unsubstituted $\beta$-1,3-linked galactan [18]. In contrast, $\beta$-1,3-galactan acceptor is approximately $25 \mathrm{kDa}$ and consists mostly of unsubstituted $\beta$-1,3-galactan (DP 154) with trace amount of $\beta$-1,6-linked Gal [29]. Galactose could be incorporated in the AGP molecule at different sites: at $O 3$ of $\beta-1,3$-galactan $\left(\beta-1,3^{\mathrm{C}}\right.$-GalT elongating $\beta-1,3-$ galactan main chain), at 06 of $\beta-1,3$-galactan $\left(\beta-1,6^{\mathrm{b}}-\right.$ GalT making 6-branches on $\beta$-1,3-galactan) and/or 06 of $\beta$-1,6galactan $\left(\beta-1,6^{\mathrm{a}}\right.$-GalT elongating $\beta-1,6$-galactan side chains; Figure 5). We investigated the site of the $\left[{ }^{14} \mathrm{C}\right]-\mathrm{Gal}$ incorporation catalyzed by the recombinant proteins among the above mentioned possibilities by treating the $\left[{ }^{14} \mathrm{C}\right]-\mathrm{Gal}$ incorporated products made onto $\mathrm{SP}_{32}$-GFP and $\beta$-1,3-galac$\tan$ with structure-specific hydrolases and subsequent size exclusion chromatography (Figure 6). The endo$\beta$-1,6-galactanase and exo- $\beta$-1,3-galactanase used in this study specifically cleave unsubstituted $\beta$-1,6-linked galactooligosaccharides of DP3 or longer [30] and $\beta-1,3-$ linked galactose regardless the presence of substitutions [31], respectively.

From the product made onto $\mathrm{SP}_{32}$-GFP, the treatment with endo- $\beta$-1,6-galactanase alone released large amounts of material eluting in the void volume, as well as small oligosaccharides with a peak at fraction 21, corresponding to DP2-3, from both the AtGALT31A/AtGALT29A complex and AtGALT29A alone (Figure 6A). The material in the void volume in Figure 6A was almost completely digested by co-treatment with endo- $\beta$-1,6-galactanase and $\alpha$-arabinofuranosidase (Figure $6 \mathrm{~B}$ ), indicating a

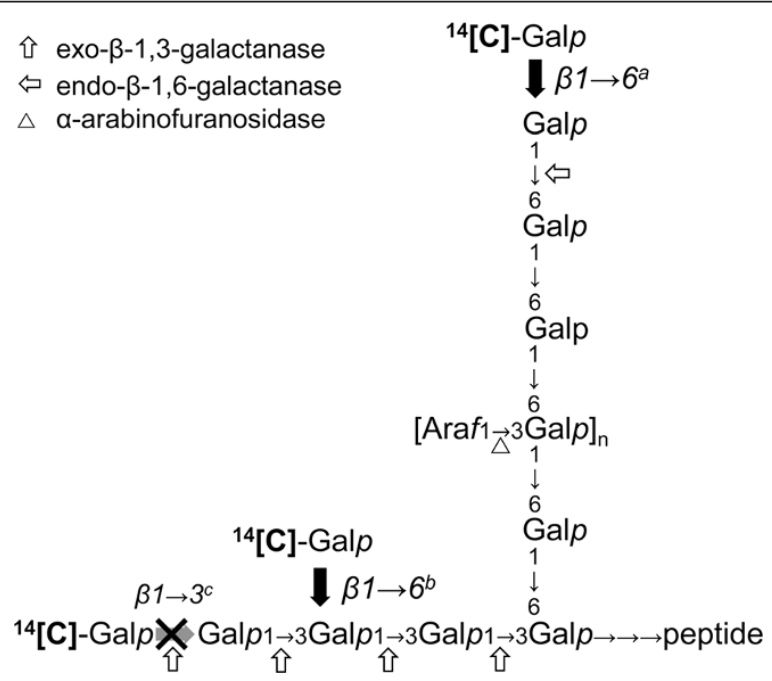

Figure 5 Simplified model structure of arabinogalactan and reaction sites of enzymes. The cleavage sites of the hydrolases (exo- $\beta-1,3$-galactanase, endo- $\beta-1,6$-galactanase, a-arabinofuranosidase) used in this paper are indicated. Recombinant AtGALT29A produced Gal incorporated products susceptible to the treatment of endo- $\beta-1,6$ - and exo- $\beta-1,3$-galactanases (Figure 6 ), therefore three possible sites $\left(\beta 1 \rightarrow 6^{a, b}\right.$ and $\beta 1 \rightarrow 3^{c}$ ) are conceivable as the candidate sites of reaction. Towards $\beta$-1,3-galactan acceptor, both $\beta 1 \rightarrow 6^{\mathrm{b}}$ and $\beta 1 \rightarrow 3^{\mathrm{c}}$ galactosyltransferase activities are possible, but the main compound released by the exo- $\beta$-1,3-galactanase treatment was galactobiose, and not galactose (inset TLC in Figure $6 C, D$ ), indicating a $\beta 1 \rightarrow 6^{b}$ activity rather than $\beta 1 \rightarrow 3^{c}$ activity. Together with the $\beta 1 \rightarrow 6^{a}$ activity indicated by the endo- $\beta-1,6$-galactanase treatment, it is concluded that, AtGALT29A possesses $\beta$-1,6-galactosyltransferase activities both on $\beta-1,3-$ and $\beta-1,6-$ galactan $\left(\beta 1 \rightarrow 6^{\mathrm{a}}\right.$, b activities). 


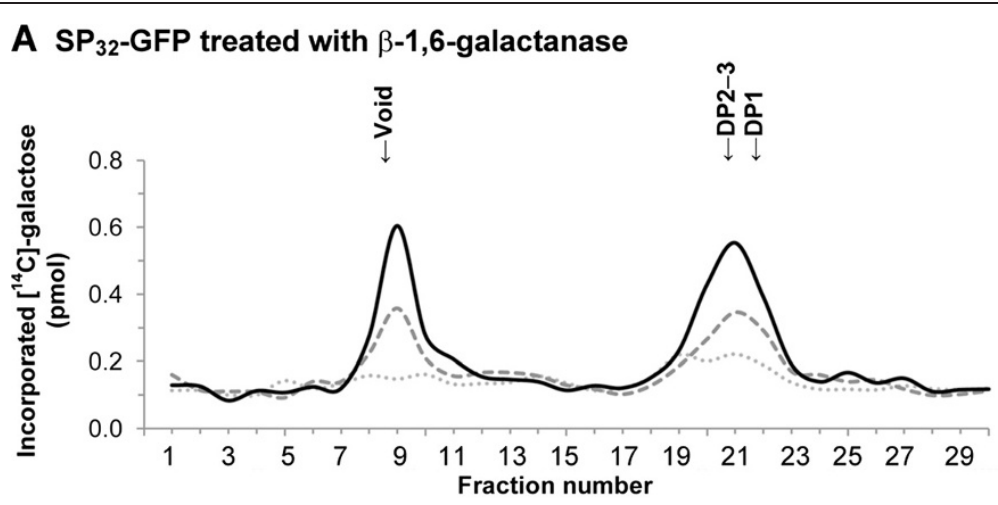

B SP 32 -GFP treated with $\beta$-1,6-galactanase $+\alpha$-arabinofuranosidase

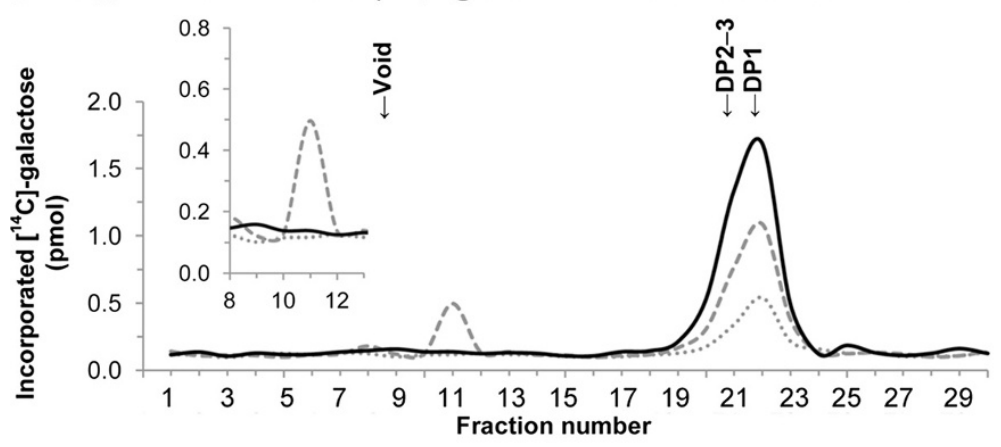

\section{C $\mathrm{SP}_{32}$-GFP treated with $\beta$-1,3-galactanase}

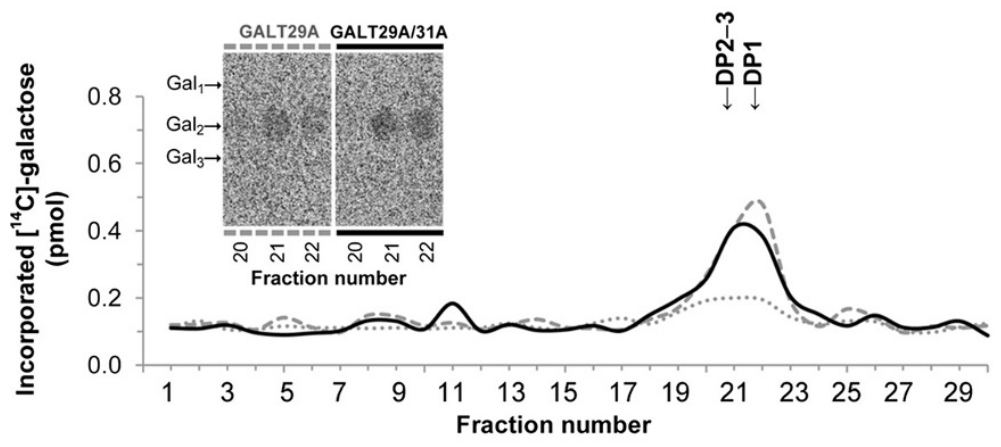

D $\beta$-1,3-galactan treated with $\beta$-1,3-galactanase

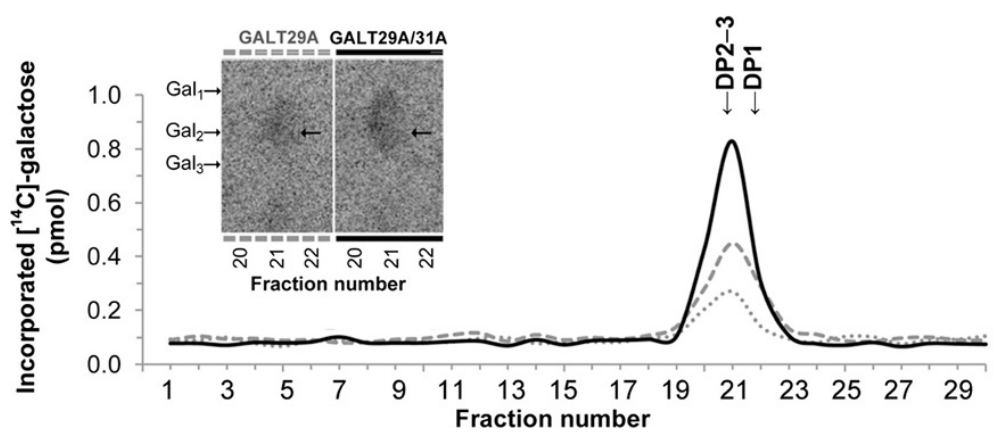




\section{(See figure on previous page.)}

Figure 6 Analysis of the sites of Gal incorporation in the products produced by AtGALT29A alone or the AtGALT29A/AtGALT31A complex.

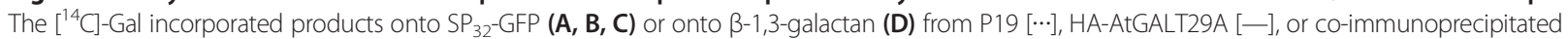
HA-AtGALT29AAtGALT31A-GFP complex [-] were treated with A: endo- $\beta$-1,6-galactanase, $\mathbf{B}$ : endo- $\beta$-1,6-galactanase + a-arabinofuranosidase, C: exo- $\beta-1,3$-galactanase, or $\mathbf{D}$ : exo- $\beta-1,3$-galactanase, and separated by size exclusion chromatography using Superdex Peptide HR 10/30. The $\left[{ }^{14} \mathrm{C}\right]$-Gal present in each fraction was evaluated by scintillation counting. Endo- $\beta-1,6-$ galactanase, $\alpha$-arabinofuranosidase, and exo- $\beta-1,3-g a l a c t a n a s e$ used in this study cleave $\beta-1,6$-linked unsubstituted galactotriose, terminal a-linked arabinofuranose, and $\beta$-1,3-linked galactooligosaccharides regardless the presence or absence of substitutions, respectively. Release of small $\left[{ }^{14} \mathrm{C}\right]$-oligosaccharides by endo- $\beta$-1,6-galactanase indicates the $\left[{ }^{14} \mathrm{C}\right]$-Gal incorporation to a part of $\beta-1,6$-galactotriose, while exo- $\beta-1,3-$ galactanase releases $\left[{ }^{14} \mathrm{C}\right]$-Gal monomer from $\beta$-1,3-linked galactan and $\left[{ }^{14} \mathrm{C}\right]$-oligosaccharide (s) from side chains attached to $\beta$-1,3-linked galactan. From the $\left[{ }^{14} \mathrm{C}\right]$-products made onto $\mathrm{SP}_{32}$-GFP and $\beta-1,3-$ galactan, exo- $\beta$-1,3-galactanase released mainly $\left[{ }^{14} \mathrm{C}\right]$-galactobiose analyzed by TLC (inset $\mathbf{C}$ and $\mathbf{D}$ ), indicating the incorporation of single $\left[{ }^{14} \mathrm{C}\right]$-Gal to $\beta$-1,3-linked Gal at $\mathrm{O} 6$ in the $\left[{ }^{14} \mathrm{C}\right]$-products. From any treatments (A-D), higher amount of small $\left[{ }^{14} \mathrm{C}\right]$-oligosaccharides are released from the $\left[{ }^{14} \mathrm{C}\right]$-products made by AtGALT29A/AtGALT31A complex compared to that from a single enzyme. The results indicate that AtGALT29A possesses $\beta-1,6-$-GalT activities elongating $\beta-1,6-$ galactan and forming 6-Gal branches on $\beta-1,3-g a l a t a n$, and the $\beta-1,6-G a l T$ activities are increased when AtGALT29A is in a protein complex with AtGALT31A.

part of $\left[{ }^{14} \mathrm{C}\right]-$ Gal incorporation occurred at the $\beta-1,6$ linked galactans substituted with Ara, and that Ara substitution sterically hindered the action of endo- $\beta$ 1,6-galactanase [30]. The results indicate that both the enzyme complex and AtGALT29A alone incorporated $\left[{ }^{14} \mathrm{C}\right]-\mathrm{Gal}$ to both Ara-substituted and non-substituted $\beta-1,6$-galactans, and the level of total Gal incorporation to both types of acceptors was much higher with AtGALT29A in a complex with AtGALT31A. AtGALT31A was previously characterized using radish AGP as acceptor for the incorporation of $\left[{ }^{14} \mathrm{C}\right]-\mathrm{Gal}$ and the product was digested by endo- $\beta$-1,6-galactanase [17]. We tested the GalT activity of AtGALT31A using $\mathrm{SP}_{32}$-GFP acceptor used in this study and showed that the level of activity of AtGALT31A alone was lower than the level observed for the AtGALT29A alone (Additional file 3: Figure S3). Hence, the overall results indicate a cooperative action of GalT activity in elongating $\beta-1,6$-galactan of type II AG by forming an enzyme complex containing AtGALT29A and AtGALT31A.

Treatment with exo- $\beta-1,3$-galactanase to the products made onto $\mathrm{SP}_{32}$-GFP released small oligosaccharides eluting at fraction 22 and 21 as a peak by AtGALT29A alone and by AtGALT29A in a complex with AtGALT31A, respectively (Figure $6 \mathrm{C}$ ). Both fractions contained galactobiose as the major component analyzed by TLC, but the amount was much higher from the product made by the AtGALT29A/AtGALT31A complex (Figure 6C, inset). Since exo- $\beta$-1,3-galactanase cleaves $\beta$-1,3-linked Gal, the detected galactobiose is likely $\beta-1,6$-linked single Gal substituted onto $\beta$-1,3-linked Gal. Thus, the results indicate that both AtGALT29A alone and the AtGALT29A/ AtGALT31A complex likely transfer Gal to $O 6$ position of $\beta$-1,3-linked galactan, and that the amounts of $\left[{ }^{14} \mathrm{C}\right]-\mathrm{Gal}$ transfer was higher by the AtGALT29A/AtGALT31A complex.

The GalT activity towards $\beta$-1,3-linked Gal was further investigated using $\beta-1,3$-galactan as acceptor (Figure 6D, [29]). When the products made on $\beta-1,3$-galactan were treated with exo- $\beta$-1,3-galactanase [31], the main peak appeared at fraction 21 (Figure 6D) and much more $\left[{ }^{14} \mathrm{C}\right]-$ Gal containing compound was released from the product made by AtGALT29A/AtGALT31A complex compared to AtGALT29A alone. The major component released was galactobiose as indicated by TLC (Figure 6D, inset) and the higher level of $\left[{ }^{14} \mathrm{C}\right]$-galactobiose was detected from the product produced by the AtGALT29A/ AtGALT31A complex, which is consistent with the result obtained from $\mathrm{SP}_{32}$-GFP analysis (Figure $6 \mathrm{C}$ ). Therefore, we confirmed that the GalT activity onto $\beta$-1,3-galactan is mainly a branch forming activity ( $\beta-1,6-$ GalT) and this activity is significantly increased by the AtGALT29A/ AtGALT31A complex compared to AtGALT29A alone.

Taken together, analysis of the enzymatic activities indicates that AtGALT29A alone has a $\beta-1,6-$ GalT activity for elongating $\beta-1,6$-galactan and forming 6-Gal branches on $\beta$-1,3-galactan, and these activities are significantly increased when AtGALT29A is in a complex with AtGALT31A.

\section{$N$. benthamiana microsomes showed increased galactose incorporation to endogenous type II AGs after co-expression of AtGALT31A and AtGALT29A}

Since in vitro analysis suggested an increase of the enzyme activity when AtGALT29A is in a complex with AtGALT31A, we also studied possible in vivo effects of co-expression of AtGALT31A and AtGALT29A for AGP glycosylation activity in $N$. benthamiana. We isolated microsomes after co-expression of both proteins and tested incorporation of exogenously added UDP- $\left[{ }^{14} \mathrm{C}\right]-$ Gal to endogenous type II AG, mediated via endogenous UDP-Gal transporter(s) and GalTs present in the lumenal side of vesicles [32]. The synthesis of type II AG products was investigated by $\left[{ }^{14} \mathrm{C}\right]-\mathrm{Gal}$ incorporated polysaccharide materials precipitated by $70 \%$ ethanol (Figure 7A), or by type II AG precipitated by the $\beta$-GalYariv reagent (Figure 7B). In both cases, the results indicated a higher level of $\mathrm{Gal}$ incorporation to the polysaccharide materials and $\beta$-Gal-Yariv precipitates 

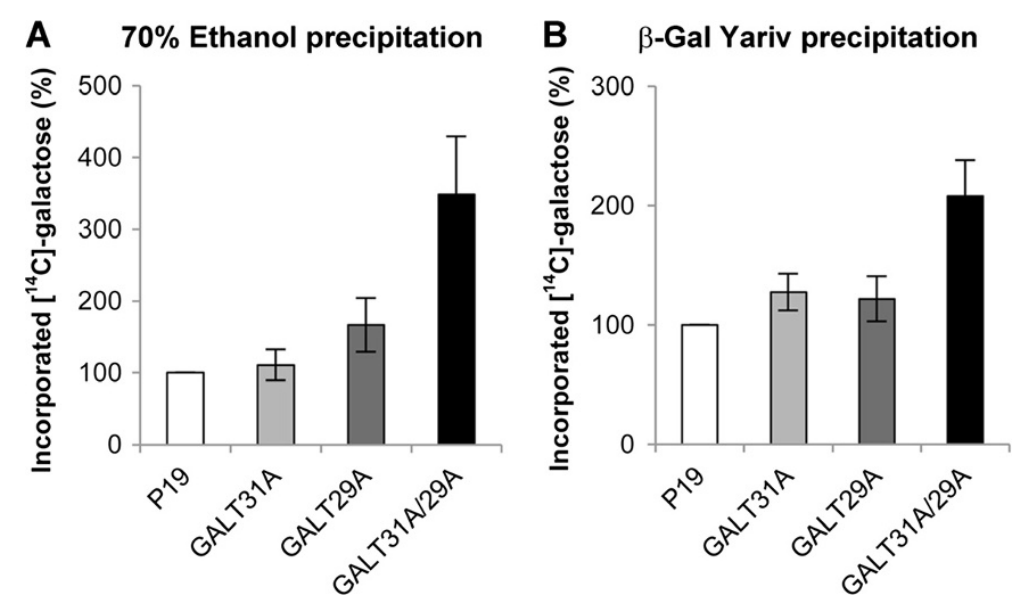

Figure 7 Galactosyltransferase activity in intact microsomes isolated from $N$. benthamiana after co-expression of HA-AtGALT29A and AtGALT31A-GFP. Microsomes were incubated with exogenously added UDP- $\left[{ }^{14} \mathrm{C}\right]$-Gal and the $\left[{ }^{14} \mathrm{C}\right]-$ Gal incorporation to luminal endogenous materials were analyzed by precipitation either by A: $70 \%$ ethanol or B: $\beta$-Gal Yariv reagent. Error bars showed standard deviations from $n=4$.

in the microsomes after co-expression of AtGALT31A and AtGALT29A compared to expression of each. Thus, the co-expression of AtGALT31A and AtGALT29A in $N$. benthamiana increases the Gal incorporation activity to endogenous type II AG materials in isolated microsomes.

\section{Discussion}

Identification of glycosyltransferases involved in the biosynthesis of type II arabinogalactan

In this paper we have shown that the protein encoded by Arabidopsis At1g08280 gene is a $\beta-1,6-$ GalT that is involved in the glycosylation of type II AG. We hypothesized that the enzyme is a putative GT involved in the biosynthesis of type II AG based on co-expression analysis together with two other GT genes previously identified in the same glycosylation pathway (AtGALT31A and AtGLCAT14A) $[17,18]$. This may appear surprising since the GT belongs to the GT29 family and the protein sequence encoded by At1g08280 contains 'sialyl motifs' conserved in sialyltransferases in mammals and fungi [20]. Sialyltransferase activity was previously tested for the protein encoded by At1g08280 and concluded to be negative [21]. Apparently the sialyl motifs do not work as independent domains, since a chimeric protein constructed with a sequence encoded by Arabidopsis At3g48820 and the sialyl motifs from human sialyltransferase did not result in sialyltransferase activity [22]. The GT29 proteins from Arabidopsis (3 proteins in Arabidopsis thaliana) and rice (5 proteins in Oryza sativa) share homologous sequences and all contain putative sialyl motifs; however, only two of the rice proteins demonstrated sialyltransferase-like activity [21], while two Arabidopsis proteins did not $[21,22]$. Thus, proteins harboring sialyl motifs apparently do not necessarily encode an enzyme with sialyltransferase activity.
It is difficult to predict the biochemical activity of putative GTs by analyzing the primary sequences, but co-expression studies based on genome-wide expression data in A. thaliana (e.g., GeneCAT) [23] were useful in identifying putative candidate GTs involved in type II AG biosynthesis. We selected AtGLCAT14A and AtGALT29A based on the co-expression profile with AtGALT31A and characterized as biosynthetic enzymes involved in type II AG glycosylation. Co-expression analysis using genes encoding the protein core for type II AG modification as markers has been established [33], which may be a good resource to investigate the rest of the pathway. In order to identify the biochemical activity of the putative GT candidates, we established screening methods to cover broad activities expected to be involved in the biosynthesis of type II AG (Figure 1). We found microsomal materials after expression of SynGMs in $N$. benthamiana quite useful for donor substrate identification as they contain a mixture of various oligosaccharides present in type II AG. Otherwise, structure-defined oligosaccharides are difficult to obtain from commercial sources, and even if available, they are expensive and only useful for a specific GT assay. Using the microsomal materials mentioned above as the acceptor mixture, we screened donor substrates for the recombinant enzyme expressed in $N$. benthamiana. The strategy worked for the characterization of AtGALT31A [17], AtGLCAT14A [18], and AtGALT29A (Figure 1), and is expected to be useful to analyze other unidentified GTs in the type II AG glycosylation pathway.

In this paper, we reported that AtGALT29A possesses $\beta-1,6-$ GalT activities for elongating $\beta$-1,6-galactan and forming 6 -Gal branches on $\beta$-1,3-galactan. Furthermore, AtGALT29A forms enzyme complex together with AtGALT31A, and the complex showed significantly 
higher level of $\beta-1,6-$ GalT activities exhibited by AtGALT29A alone.

\section{Impact of the protein complexes in the glycosylation processes}

Based mainly on the studies using yeast and mammalian enzymes, evidence of protein-protein interactions among GTs has been accumulated, namely, that several GTs can form homomeric complexes with themselves and/or interact with other GTs or non-GT proteins via heteromeric complexes (for review see [34]). The complex formation is considered to serve various biological significances, e.g., activate/stabilize the catalytic activity, alternate the substrate specificity, allow proper targeting, and control the localization in ER/Golgi apparatus. In addition, the clusters of GTs are considered to be an assembly line for the efficient and accurate production of certain glycoforms by substrate channeling (for reviews see [35,36]). In plants, evidence for protein-protein interactions between GTs in the secretory pathway are emerging for the biosynthesis of pectin (GAUT1 and GAUT7 [37], (ARAD1 and ARAD2 [38]), xyloglucan (CSLC4, XXT1/XXT2, and XXT5) $[39,40]$, glucuronoarabinoxylan (IRX10 and IRX14) [41], and protein $N$-glycosylation (GMI, GnTI, GMII and XylT) [42]. A putative interaction is also implicated from the cooperative activity and/or co-expression profile in the biosynthesis of galactomannan (ManS and GMGT) [43], xylan (IRX9 and IRX14) [44,45] and mannan (CSLD2 and CSLD3) [46]. The interaction of GAUT1 to GAUT7 has been demonstrated to be important to target catalytic domain of GAUT1 to the Golgi [37], but besides this study, little is known for the significance of forming protein complex(es) among GTs in plants.

In this paper, we evidently demonstrate the presence of homodimeric interactions between for both, AtGALT29A and AtGALT31A by FRET analysis, and do this also for heterodimeric ones between AtGALT31A and AtGALT29A, when these proteins were ectopically expressed in $N$. benthamiana leaves (Figure 3). Moreover, AtGALT31A-YFP could biochemically be co-immunoprecipitated using HA antibody against $\mathrm{HA}$ epitope tagged $\mathrm{N}$-terminally to AtGALT29A (Figure 4), and the protein complex(es) containing AtGALT31A-YFP and HA-AtGALT29A exhibited an increased level of $\beta-1,6$-GalT activities compared to HA-AtGALT29A alone (Figure 6). Therefore, the complex formation may have a regulatory role in the $\beta-1,6$-galactan biosynthesis in type II AG. Accordingly, the present study offers one of the few examples showing a biological significance in the molecular interaction between GTs in plants. It is conceivable that the regulation of biosynthesis via formation of protein complexes among biosynthetic enzymes is faster than transcriptional regulation, and that this mode allows determining subtle changes of cell-surface type
II AG structures during cell differentiation in plants. How common such a system for other GTs involved in the biosynthesis of type II AG remains to be elucidated.

According to different levels of FRET efficiencies among different combination of AtGLAT29A and AtGLAT31A, tagged with mCER3 and YFP and reciprocally, respectively, we suggest that AtGALT31A is less capable of dimerization, while AtGALT29A forms dimers more effectively than AtGALT31A. Furthermore, formation of heterodimers between AtGALT31A and AtGALT29A seems to be more dominant than that of homodimers when both AtGALT31A and AtGALT29A are available. With increasing probability we suggest occurrence of dimerization in following sequence: AtGALT31A monomer, AtGALT31A homodimer, AtGALT29A homodimer, and finally AtGALT31A/AtGALT29A heterodimer.

Since the FRET efficiencies might be influenced by the protein stoichiometry in the Golgi stacks, we tried to quantify the proteins expressed ectopically in $N$. benthamiana, but failed because of the low level of protein expression. We could not detect the expressed proteins in $N$. benthamiana microsomes analyzed by SDS-PAGE followed by Western blot. Neither did Native-PAGE lead to detectable amounts in Western blots (data not shown). Therefore we could neither normalize the FRET efficiencies based on the protein concentration nor detect protein complexes under the experimental condition used. However, acceptor photobleaching, which is the method used for calculating the FRET efficiencies in the present study, is quite robust against differences in expression of the two FRET partners, when compared to sensitized emission [26]. Eventually, immunoprecipitation of the proteins in microsomes from $N$. benthamiana allowed us to detect the recombinant proteins by Western blot analysis (Figure 4).

\section{Conclusions}

The AtGALT29A (At1g08280) from Arabidopsis thaliana encodes a $\beta-1,6$-GalT involved in the biosynthesis of type II AG by heterologous expression of the protein in $N$. benthamiana and the biochemical enzyme assay. When expressed simultaneously, AtGALT29A interacted with AtGALT31A, and the enzyme complex exhibited substantially increased level of $\beta-1,6-$ GalT activities compared to AtGALT29A alone. The complex formation could be an important regulatory mechanism for producing $\beta$-1,6-galactan side chains of type II AG during plant development.

\section{Methods}

\section{Materials}

Full-length At1g08280 cDNA with and without stop codon cloned into the Gateway vector, pDONR221 and pDONR223, respectively, were the kind gifts of 
Dr. Masood Z. Hadi (Joint BioEnergy Institute, Lawrence Berkeley National Laboratory). Plasmids encoding synthetic glycomodule peptides of AGP in a pBI121 vector (SynGMs: GAGP $_{8}$ and $\mathrm{SP}_{32}$ ) $[24,28]$ were the kind gifts of Dr. Marcia Kieliszewski (Ohio University). Preparation of endo- $\beta$-1,6-galactanase from Streptomyces avermitilis (Sa1,6Gal5A) [30] and exo- $\beta$-1,3-galactanase from Phanerochaete chrysosporium (Pc1,3Gal43A) [31] followed the procedure described in the publications. Radiochemicals were from PerkinElmer (Boston, MA). UDP-Xyl was from CarboSource (Complex Carbohydrate Resource Center), and other nucleoside diphosphate (NDP) sugars were from Calbiochem-Novabiochem. Other chemicals were from Sigma-Aldrich unless otherwise specified.

\section{DNA constructions}

For enzyme assays, full-length At1g08280 cDNA containing a stop codon cloned in pDONR221 was moved into pEarleyGate 201 vector [47] to create a hemagglutinin (HA) fusion tag at the N-terminus using LR clonase II (Invitrogen, Life Technologies, Carlsbad, CA). Generation of a C-terminal GFP fusion construct for AtGALT31A (At1g32930) in the pGWB6 vector is described in [17]. For microscope analyses, full-length cDNA sequences without a stop codon cloned in pDONR223 were moved into a modified pEarleyGate vector containing monomeric CFP (pEarleyGate mCer3; vector construction as described in [18]) and pEarleyGate 101 [48] to generate C-terminal mCer3-HA and YFP-HA fusions, respectively. Expression constructs were transformed into Agrobacterium tumefaciens strain C58C1 pGV3850 for expression in N. benthamiana. Full-length At5g39990 (AtGLCAT14A) [18] cDNA containing a stop codon cloned in pDONR221 was moved into pEarleyGate 201 vector as described above.

\section{Expression of recombinant proteins in N. benthamiana} Infiltration of $N$. benthamiana leaves with Agrobacterium strain(s) harboring the appropriate GT(s) was always performed as co-infiltration with the strain harboring the p19 construct as described in [17]. The p19 protein derived from tomato bushy stunt virus works as a suppressor of gene silencing in the Agrobacterium-mediated transient gene expression system [49]. For enzyme assays, $N$. benthamiana leaves were co-infiltrated with Agrobacterium strains at a final cell density of $\mathrm{OD}_{600}=0.4$. For the negative control, only the Agrobacterium strain harboring the $p 19$ construct at a cell density of $\mathrm{OD}_{600}=$ 0.2 was infiltrated. The infiltrated plants were grown in a greenhouse $\left(28^{\circ} \mathrm{C} /\right.$ day, $25^{\circ} \mathrm{C} /$ night with a $16 \mathrm{~h}$ photoperiod) and harvested at 4 days post-infiltration. For microscope analyses, $N$. benthamiana leaves were co-infiltrated using the procedure described in [38] with Agrobacterium strains at a final cell density of
$\mathrm{OD}_{600}=0.5$. The infiltrated plants were grown in a growth chamber $\left(25^{\circ} \mathrm{C}\right.$ with $16 \mathrm{~h}$ photoperiod, $70 \% \mathrm{hu}$ midity) for 50 hours prior to analysis.

\section{Purification of recombinant enzymes and enzyme complexes}

Preparation of the microsome after expression of the recombinant enzymes followed the procedure described in [17]. The total protein concentration of microsome solutions was adjusted to $5 \mu \mathrm{g} / \mu \mathrm{L}$ and treated with $n$-dodecyl $\beta$-maltoside (final concentration of $5 \mathrm{mM}$ ). To affinity purify the GFP fusion proteins, detergent-treated microsomal membranes (1 $\mathrm{mg}$ total protein) was incubated with $0.8 \mu \mathrm{g}$ anti-GFP from mouse $\mathrm{IgG}_{1} \mathrm{~K}$ (Roche Diagnostics, Indianapolis, IN) at $4^{\circ} \mathrm{C}$ for $2-3 \mathrm{~h}$ with rotation followed by addition of $20 \mu \mathrm{L}$ of protein $\mathrm{G}$ agarose slurry (contain $50 \%$ resin, pre-equilibrated in PBS) and additional incubation overnight at $4^{\circ} \mathrm{C}$. For HA affinity purification, detergent-treated microsomal membranes $(1 \mathrm{mg}$ total protein) was incubated with $20 \mu \mathrm{L}$ of monoclonal antiHA agarose slurry (containing 50\% resin) equilibrated in PBS with rotation for overnight at $4^{\circ} \mathrm{C}$. In both treatments, the enzyme-immobilized resin was collected by centrifugation at $500 \times \mathrm{g}, 30 \mathrm{sec}$, at $4^{\circ} \mathrm{C}$ followed by three washing steps in PBS. The enzymeimmobilized resin was suspended in an equal volume of 50 mM HEPES, pH 7.0 with 10\% glycerol [17] and used immediately for enzyme assay.

\section{Preparation of AG acceptors (GAGP ${ }_{8}$-GFP, $\mathrm{SP}_{32}$-GFP, and $\beta$-1,3-galactan)}

Preparation of the microsome after expression of AG glycopeptides (SynGMs; GAGP ${ }_{8}$-GFP and $\mathrm{SP}_{32}$-GFP), is described in [18]. The polysaccharide analysis using carbohydrate gel electrophoresis (PACE) after digestion with the specific exo- $\beta-1,3$-galactanase indicated very similar compositions derived from type II AG for the $\mathrm{SP}_{32}$-GFP material and $\mathrm{GAGP}_{8}$-GFP used previously [17], indicating the presence of $\beta$-1,6-galactooligosaccharides with DP 1 to 8 , which are partially decorated with Ara, and the presence of unsubstituted main chain $\beta$-1,3-galac$\tan$ for both types of acceptors. $\beta-1,3-$ Galactan was prepared by three times Smith degradation of Gum arabic [29], which contains mainly $\beta$-1,3-linked Gal and a trace amount of $\beta-1,6$-linked Gal. Average molecular weight is around $25 \mathrm{kDa}$, which corresponds to DP of ca. 154.

\section{Enzyme assay}

The enzyme assays substantially followed the methods described in [17]. For identification of the donor-substrate, the reaction was performed in the presence of combined or individual NDP-sugars as described in $[17,18]$. The reaction was performed in the presence of $0.1 \mathrm{mM} \mathrm{NDP-sugar}$ (containing $277.5 \mathrm{~Bq}$ of $\mathrm{NDP}-\left[{ }^{14} \mathrm{C}\right.$-]-sugar), $28 \mathrm{mM}$ 
HEPES, $10 \mathrm{mM} \mathrm{MnCl} 2$, pH 7.0, and $5 \mu \mathrm{L}$ of enzymeimmobilized resin and $5 \mu \mathrm{L}$ of GAGP $_{8}-$ GFP $(5 \mu \mathrm{g} / \mu \mathrm{L})$ as the acceptor. The reaction was performed at $22^{\circ} \mathrm{C}$ for $16 \mathrm{~h}$ and the products were precipitated in the presence of $0.25 \mu \mathrm{L}$ of $10 \mathrm{mg} / \mathrm{ml}$ horseradish peroxidase and $0.28 \mu \mathrm{L}$ of $0.3 \% \mathrm{H}_{2} \mathrm{O}_{2}$ [47]. The presence of $\left[{ }^{14} \mathrm{C}\right]$-sugars in the pellet was determined by scintillation counting after washing several times with water.

In case the product was further analyzed by hydrolases, the reaction was performed in the presence of higher amount of UDP- $\left[{ }^{14} \mathrm{C}\right]$-Gal, using $5 \mu \mathrm{L}$ of enzymeimmobilized resin with $5 \mu \mathrm{L}$ of $\mathrm{SP}_{32}$-GFP $(5 \mu \mathrm{g} / \mu \mathrm{L})$ or $4 \mu \mathrm{L}$ of $\beta$-1,3-galactan $(1 \mathrm{mM})$ in the presence of $1480 \mathrm{~Bq}$ UDP- $\left[{ }^{14} \mathrm{C}\right]$-Gal, $28 \mathrm{mM}$ HEPES, $10 \mathrm{mM} \mathrm{MnCl}$, $\mathrm{pH} 7.0$ in a total assay volume of $25 \mu \mathrm{L}$.

The enzyme assay using intact microsomes followed the method described in [34] in a total assay volume of $25 \mu \mathrm{L}$. After $1 \mathrm{~h}$ incubation at $25^{\circ} \mathrm{C}, 250 \mu \mathrm{L}$ of water was added and the mixture was sonicated for $10 \mathrm{sec}$ to burst the microsomal vesicles. $\left[{ }^{14} \mathrm{C}\right]$-incorporated products were precipitated either by $70 \%(\mathrm{v} / \mathrm{v})$ ethanol at $-20^{\circ} \mathrm{C}$ for $30 \mathrm{~min}$ or $\beta$-galactosyl Yariv reagent $(10 \mu \mathrm{L}$ of $10 \mathrm{mg} / \mathrm{mL} \beta$-Gal-Yariv in the presence of $150 \mathrm{mM} \mathrm{NaCl}$, Biosupplies) at $4^{\circ} \mathrm{C}$ overnight. The precipitated materials were collected by centrifugation at $10,000 \times \mathrm{g}, 12^{\circ} \mathrm{C}$ for 15 min followed by washing three times with $70 \%$ ethanol or $150 \mathrm{mM} \mathrm{NaCl}$ prior to scintillation counting.

\section{Product analysis}

The products made onto $\mathrm{SP}_{32}$-GFP acceptor were collected by incubating with $1 \mu \mathrm{L}$ anti-GFP monoclonal antibody (Roche) for overnight at $4{ }^{\circ} \mathrm{C}$. An additional $10 \mu \mathrm{L}$ of protein G-agarose slurry (containing 50\% resin) in PBS was added and incubated at $22^{\circ} \mathrm{C}$ for $1.5 \mathrm{~h}$ with rotation. Immunoprecipitated material was collected by centrifugation at $200 \times \mathrm{g}$ for $30 \mathrm{sec}$ at $4^{\circ} \mathrm{C}$ followed by washing three times with $150 \mathrm{mM} \mathrm{NaCl}$. The product made onto $\beta$-1,3-galactan was precipitated in $70 \%$ ethanol and washed three times with $70 \%$ ethanol. Treatments with $0.0022 \mathrm{U}$ endo- $\beta-1,6$-galactanase and $0.02 \mathrm{U}$ exo- $\beta-1,3$-galactanase in $80 \mathrm{mM}$ Mcllvaine buffer at pH 5.5 and 4.5, respectively, are described in [17]. Cotreatment of the product with $\alpha$-arabinofuranosidase $(0.08$ $\mathrm{U}$, Megazyme) was performed in $80 \mathrm{mM}$ McIlvaine buffer at $\mathrm{pH} 5.5$, together with $0.0022 \mathrm{U}$ endo- $\beta$-1,6-galactanase. The hydrolyzed products were applied to a Superdex Peptide HR 10/30 column (GE Healthcare) and eluted by $50 \mathrm{mM}$ ammonium formate (flow rate: $0.4 \mathrm{~mL} / \mathrm{min}$, $2 \mathrm{~min} /$ fraction). The $\left[{ }^{14} \mathrm{C}\right]$-sugars in the fractions were analyzed by scintillation counting.

Thin layer chromatography (TLC) was performed by the samples developed with acetonitrile/water $(80: 20, \mathrm{v} / \mathrm{v})$ onto the TLC plate (Silica gel $60 \mathrm{~F}_{254}$; Merck, Darmstadt, Germany). Carbohydrate standards were visualized by
$\mathrm{H}_{2} \mathrm{SO}_{4} /$ ethanol (10:90, v/v) followed by charring at $120^{\circ} \mathrm{C}$ and the $\left[{ }^{14} \mathrm{C}\right]$-Gal was detected using a Phosphor-Imager (Molecular Dynamics Storm 860; GE Healthcare).

\section{Protein analyses}

Determination of the protein concentration, SDS-PAGE and western blotting are described in [18]. Native-PAGE was performed by NativePage Bis-Tris Gel System according to the manufacture (Invitrogen, Life Technologies, Carlsbad, CA).

\section{Subcellular localization and acceptor photobleaching FRET}

After infiltration with Agrobacterium harboring appropriate constructs, epidermal cell layers of $N$. benthamiana were analyzed by the method described in $[26,27]$. The following corrections were used: background subtraction, correction for donor photobleaching during the acquisition cycle (in the range of 1-3\%), correction for acceptor cross talk into the donor channel (1-6\%), correction for acceptor photoproduct formed upon bleaching (0.5-5\%), and correction for the incomplete photobleaching of the acceptor (in the range of 10-40\% unbleached fraction). Regions of interest (ROIs) representing Golgi vesicles were segmented as described in [26], and rejected from further analysis if (1) their size was below 4 squarepixels, (2) circularity below 0.3 , (3) the percentage of pixels above background in the ROI changed by more than $30 \%$ in the post-bleach image, (4) over $30 \%$ of their pixels showed out-of-range FRET efficiency, and (5) their averaged FRET efficiency was below -0.05 . The pixel-by-pixel distribution of FRET efficiency for each protein combination was generated from pooling all valid ROIs.

\section{Additional files}

Additional file 1: Figure S1. Co-expression analysis of AtGALT29A, AtGALT31A and AtGICAT14A.

Additional file 2: Figure S2. Localization and FRET analysis for AtGALT29A and AtGLCAT14A.

Additional file 3: Figure S3. Analysis of the products made onto $\mathrm{SP}_{32}$-GFP by P19 only control or AtGALT31A.

\section{Abbreviations}

AGP: Arabinogalactan protein; Ara: $\alpha$-L-arabinofuranoside; AtGALT29A: Arabidopsis thaliana $\beta$-galactosyltransferase 1 from family GT29 (At1g08280); AtGALT31A: A. thaliana $\beta$-galactosyltransferase 1 from family GT31 (At1g32930); AtGlCAT14A: A. thaliana $\beta$-glucuronosyltransferase 1 from family GT14 (At5g39990); Fuc: Fucose; GAGP: synthetic glycomodule gene harbouring 8 repetitive 19-residue consensus motif of gum Arabic glycoprotein; Gal: Galactose; GalT: Galactosyltransferase; Glc: Glucose; GlcNAc: N-acetyl-Dglucosamine; GlcA: Glucuronic acid; GT: Glycosyltransferase; HA: Hemagglutinin; Man: Mannose; mCer3: Monomeric Cerulean3; NDP: Nucleoside diphosphate; PACE: Polysaccharide analysis using carbohydrate gel electrophoresis; Rha: Rhamnose; ROI: Region of interest; type II AG: arabino- $\beta-1,3-(\beta-1,6)$-galactan; SD: Standard deviation; $\mathrm{SP}_{32}$ : Synthetic glycomodule gene harbouring 32 repeats of the Ser-Pro motif; $\mathrm{ST}_{\mathrm{tmd}^{-}}$ YFP: Sialyltransferase short cytoplasmic tail and single transmembrane 
domain fused to YFP; SynGM: Synthetic glycopeptide/glycomodule; Xyl: Xylose.

\section{Competing interests}

The authors declare that they have no competing interests.

\section{Authors' contributions}

$A D$ and CPP contributed substantially to design the experiments, to perform the experiments and drafted the manuscript. In particular, AD contributed to the biochemical enzyme assays, purification of the protein complex and its analysis. CPP contributed to the study of subcellular localization and FRET based protein-protein interaction of glycosyltransferases. GV supervised experimental design and data analysis of the FRET acceptor photobleaching study. SK prepared oligosaccharides and specific hydrolases used for the biochemical enzyme assays. AS supervised the use of confocal laser scanning microscopy and guided the FRET analysis. NG conceived and coordinated the project and wrote the manuscript. All authors read and approved the final manuscript.

\section{Acknowledgements}

This research was supported by the Danish Council for Strategic Research, Food, Health and Welfare [09-067059] and the Danish Council for Independent Research, Technology and Production Sciences [274-09-0113] to NG. We would like to thank Drs. Paul Dupree and Theodra Tryfona for structural analysis of the SynGM acceptors. Imaging data were collected at the Center for Advanced Bioimaging (CAB) Denmark, University of Copenhagen.

\section{Author details}

${ }^{1}$ Department of Plant and Environmental Sciences, Thorvaldsensvej 40, 1871 Frederiksberg, C, Denmark. ${ }^{2}$ Department of Biophysics and Cell Biology, and MTA-DE Cell Biology and Signaling Research Group, University of Debrecen, Debrecen, Hungary. ${ }^{3}$ Food Biotechnology Division, National Food Research Institute, 2-1-12 Kannondai, Tsukuba, Ibaraki 305-8642, Japan. ${ }^{4}$ Present address: Fungal Physiology, CBS-KNAW, Fungal Biodiversity Center, Uppsalalaan 8, Utrecht 3584, CT, The Netherlands.

\section{Received: 13 November 2013 Accepted: 25 March 2014}

Published: 3 April 2014

\section{References}

1. Seifert GJ, Roberts K: The biology of arabinogalactan proteins. In Annual Review of Plant Biology, Volume 58. Palo Alto: Annual Reviews; 2007:137-161.

2. Pennell Rl, Janniche L, Kjellbom P, Scofield GN, Peart JM, Roberts K: Developmental Regulation of a Plasma-Membrane Arabinogalactan Protein Epitope in Oilseed Rape Flowers. Plant Cell 1991, 3(12):1317-1326.

3. Pennell RI, Knox JP, Scofield GN, Selvendran RR, Roberts K: A Family of Abundant Plasma-Membrane Associated Glycoproteins Related to the Arabinogalactan Proteins Is Unique to Flowering Plants. J Cell Biol 1989, 108(5):1967-1977.

4. Stacey NJ, Roberts K, Carpita NC, Wells B, McCann MC: Dynamic changes in cell surface molecules are very early events in the differentiation of mesophyll cells from Zinnia elegans into tracheary elements. Plant $J$ 1995, 8(6):891-906.

5. Stacey NJ, Roberts K, Knox JP: Patterns of Expression of the Jim4 Arabinogalactan-Protein Epitope in Cell-Cultures and during Somatic Embryogenesis in Daucus-Carota L. Planta 1990, 180(2):285-292.

6. Casero PJ, Casimiro I, Knox JP: Occurrence of cell surface arabinogalactan-protein and extensin epitopes in relation to pericycle and vascular tissue development in the root apex of four species. Planta 1998, 204(2):252-259.

7. Dolan L, Linstead P, Roberts K: An AGP epitope distinguishes a central metaxylem initial from other vascular initials in the Arabidopsis root. Protoplasma 1995, 189(3-4):149-155.

8. Dolan L, Roberts K: Secondary Thickening in Roots of Arabidopsis-Thaliana Anatomy and Cell-Surface Changes. New Phytol 1995, 131(1):121-128.

9. Knox JP, Linstead PJ, Peart J, Cooper C, Roberts K: Developmentally Regulated Epitopes of Cell-Surface Arabinogalactan Proteins and Their Relation to Root-Tissue Pattern-Formation. Plant J 1991, 1(3):317-326.

10. Pennell Rl, Roberts K: Sexual Development in the Pea Is Presaged by Altered Expression of Arabinogalactan Protein. Nature 1990, 344(6266):547-549.
11. Schindler T, Bergfeld R, Schopfer P: Arabinogalactan Proteins in Maize Coleoptiles - Developmental Relationship to Cell-Death during Xylem Differentiation but Not to Extension Growth. Plant J 1995, 7(1):25-36.

12. Ellis M, Egelund J, Schultz CJ, Bacic A: Arabinogalactan-proteins: Key regulators at the cell surface? Plant Physiol 2010, 153(2):403-419.

13. Tan L, Varnai P, Lamport DTA, Yuan CH, Xu JF, Qiu F, Kieliszewski MJ: Plant $O$-hydroxyproline arabinogalactans are composed of repeating trigalactosyl subunits with short bifurcated side chains. J Biol Chem 2010, 285(32):24575-24583.

14. Tryfona T, Liang HC, Kotake T, Tsumuraya $Y$, Stephens E, Dupree $P$ : Structural characterization of Arabidopsis leaf arabinogalactan polysaccharides. Plant Physiol 2012, 160(2):653-666.

15. Wu YY, Williams M, Bernard S, Driouich A, Showalter AM, Faik A: Functional identification of two nonredundant Arabidopsis alpha(1,2)

fucosyltransferases specific to arabinogalactan proteins. J Biol Chem 2010, 285(18):13638-13645.

16. Basu D, Liang Y, Liu X, Himmeldirk K, Faik A, Kieliszewski M, Held M, Showalter AM: Functional Identification of a hydroxyproline-Ogalactosyltransferase specific for arabinogalactan protein biosynthesis in Arabidopsis. J Biol Chem 2013, 288(14):10132-10143.

17. Geshi N, Johansen JN, Dilokpimol A, Rolland A, Belcram K, Verger S, Kotake T, Tsumuraya Y, Kaneko S, Tryfona T, Dupree P, Scheller HV, Hofte $\mathrm{H}$, Mouille $\mathrm{G}$ : A galactosyltransferase acting on arabinogalactan protein glycans is essential for embryo development in Arabidopsis. Plant J 2013, 76(1):128-137.

18. Knoch E, Dilokpimol A, Tryfona T, Poulsen CP, Xiong GY, Harholt J, Petersen BL, Ulvskov P, Hadi MZ, Kotake T, Tsumuraya Y, Pauly M, Dupree P, Geshi N: A beta-glucuronosyltransferase from Arabidopsis thaliana involved in biosynthesis of type II arabinogalactan has a role in cell elongation during seedling growth. Plant J 2013, 76(6):1016-1029.

19. Cantarel BL, Coutinho PM, Rancurel C, Bernard T, Lombard V, Henrissat B: The Carbohydrate-Active EnZymes database (CAZy): an expert resource for Glycogenomics. Nucleic Acids Res 2009, 37:D233-D238.

20. Audry M, Jeanneau C, Imberty A, Harduin-Lepers A, Delannoy P, Breton C: Current trends in the structure-activity relationships of sialyltransferases. Glycobiology 2011, 21(6):716-726.

21. Takashima S, Abe T, Yoshida S, Kawahigashi H, Saito T, Tsuji S, Tsujimoto M: Analysis of sialyltransferase-like proteins from Oryza sativa. J Biochem 2006, 139(2):279-287.

22. Daskalova SM, Pah AR, Baluch DP, Lopez LC: The Arabidopsis thaliana putative sialyltransferase resides in the Golgi apparatus but lacks the ability to transfer sialic acid. Plant Biol 2009, 11(3):284-299.

23. Mutwil M, Obro J, Willats WGT, Persson S: GeneCAT - novel webtools that combine BLAST and co-expression analyses. Nucleic Acids Res 2008, 36:W320-W326.

24. Xu JF, Shpak E, Gu TY, Moo-Young M, Kieliszewski M: Production of recombinant plant gum with tobacco cell culture in bioreactor and gum characterization. Biotechnol Bioeng 2005, 90(5):578-588

25. Boevink P, Oparka K, Cruz SS, Martin B, Betteridge A, Hawes C: Stacks on tracks: the plant Golgi apparatus traffics on an actin/ER network. Plant $J$ 1998, 15(3):441-447.

26. Poulsen CP, Vereb G, Geshi N, Schulz A: Inhibition of cytoplasmic streaming by cytochalasin $D$ is superior to paraformaldehyde fixation for measuring FRET between fluorescent protein-tagged Golgi components. Cytom Part A 2013, 83(9):830-838.

27. Roszik J, Szollosi J, Vereb G: AccPbFRET: An ImageJ plugin for semi-automatic, fully corrected analysis of acceptor photobleaching FRET images. BMC Bioinformatics 2008, 9

28. Shpak E, Leykam JF, Kieliszewski MJ: Synthetic genes for glycoprotein design and the elucidation of hydroxyproline-O-glycosylation codes. Proc Natl Acad Sci U S A 1999, 96(26):14736-14741.

29. Sekimata M, Ogura K, Tsumuraya Y, Hashimoto Y, Yamamoto S: A beta-galactosidase from radish (Raphanus sativus I) seeds. Plant Physiol 1989, 90(2):567-574.

30. Ichinose $H$, Kotake T, Tsumuraya $Y$, Kaneko S: Characterization of an endo-beta-1,6-galactanase from Streptomyces avermitilis NBRC14893. Appl Environ Microbiol 2008, 74(8):2379-2383.

31. Ichinose $H$, Yoshida M, Kotake $T$, Kuno A, Igarashi $K$, Tsumuraya $Y$, Samejima M, Hirabayashi J, Kobayashi H, Kaneko S: An exo-beta-1,3-galactanase having a novel beta-1,3-galactan-binding module from Phanerochaete chrysosporium. J Biol Chem 2005, 280(27):25820-25829. 
32. Geshi N, Jorgensen B, Scheller HV, Ulvskov P: In vitro biosynthesis of 1,4-betagalactan attached to rhamnogalacturonan I. Planta 2000, 210(4):622-629.

33. Showalter AM, Keppler B, Lichtenberg J, Gu DZ, Welch LR: A Bioinformatics approach to the identification, classification, and analysis of hydroxyproline-rich glycoproteins. Plant Physiol 2010, 153(2):485-513.

34. Seko A: Complex formation of glycosyltransferases and their biological significance. Trends Glycosci Glycotechnol 2006, 18(101):209-230.

35. de Graffenried CL, Bertozzi CR: The roles of enzyme localisation and complex formation in glycan assembly within the Golgi apparatus. Curr Opin Cell Biol 2004, 16(4):356-363.

36. Young WW: Organization of Golgi glycosyltransferases in membranes: Complexity via complexes. J Membr Biol 2004, 198(1):1-13.

37. Atmodjo MA, Sakuragi Y, Zhu X, Burrell AJ, Mohanty SS, Atwood JA, Orlando R, Scheller HV, Mohnen D: Galacturonosyltransferase (GAUT) 1 and GAUT7 are the core of a plant cell wall pectin biosynthetic homogalacturonan: galacturonosyltransferase complex. Proc Natl Acad Sci U S A 2011, 108(50):20225-20230.

38. Harholt J, Jensen JK, Verhertbruggen Y, Sogaard C, Bernard S, Nafisi M, Poulsen CP, Geshi N, Sakuragi Y, Driouich A, Knox JP, Scheller HV: ARAD proteins associated with pectic Arabinan biosynthesis form complexes when transiently overexpressed in planta. Planta 2012, 236(1):115-128.

39. Chou YH, Pogorelko G, Zabotina OA: Xyloglucan xylosyltransferases XXT1, XXT2, and XXT5 and the glucan synthase CSLC4 form Golgi-localized multiprotein complexes. Plant Physiol 2012, 159(4):1355-1366.

40. Cocuron JC, Lerouxel O, Drakakaki G, Alonso AP, Liepman AH, Keegstra K, Raikhel N, Wilkerson CG: A gene from the cellulose synthase-like C family encodes a beta-1,4 glucan synthase. Proc Natl Acad Sci U S A 2007, 104(20):8550-8555.

41. Zeng W, Jiang N, Nadella R, Killen TL, Nadella V, Faik A: A Glucurono (arabino)xylan synthase complex from wheat contains members of the GT43, GT47, and GT75 families and functions cooperatively. Plant Physiol 2010, 154(1):78-97.

42. Schoberer J, Liebminger E, Botchway SW, Strasser R, Hawes C: Time-resolved fluorescence imaging reveals differential interactions of $\mathrm{N}$-glycan processing enzymes across the Golgi stack in planta. Plant Physiol 2013, 161(4):1737-1754.

43. Edwards ME, Marshall E, Gidley MJ, Reid JSG: Transfer specificity of detergent-solubilized fenugreek galactomannan galactosyltransferase. Plant Physiol 2002, 129(3):1391-1397.

44. Lee C, Teng Q, Huang WL, Zhong RQ, Ye ZH: The Arabidopsis family GT43 glycosyltransferases form two functionally nonredundant groups essential for the elongation of glucuronoxylan backbone. Plant Physiol 2010, 153(2):526-541.

45. Wu AM, Hornblad E, Voxeur A, Gerber L, Rihouey C, Lerouge P, Marchant A: Analysis of the Arabidopsis IRX9/IRX9-L and IRX14/IRX14-L pairs of glycosyltransferase genes reveals critical contributions to biosynthesis of the hemicellulose glucuronoxylan. Plant Physiol 2010, 153(2):542-554.

46. Yin L, Verhertbruggen Y, Oikawa A, Manisseri C, Knierim B, Prak L, Jensen JK, Knox JP, Auer M, Willats WGT, Scheller HV: The cooperative activities of CSLD2, CSLD3, and CSLD5 are required for normal Arabidopsis development. Mol Plant 2011, 4(6):1024-1037.

47. Kjellbom P, Snogerup L, Stohr C, Reuzeau C, McCabe PF, Pennell Rl: Oxidative cross-linking of plasma membrane arabinogalactan proteins. Plant J 1997, 12(5):1189-1196.

48. Earley KW, Haag JR, Pontes O, Opper K, Juehne T, Song KM, Pikaard CS: Gateway-compatible vectors for plant functional genomics and proteomics. Plant J 2006, 45(4):616-629.

49. Voinnet O, Rivas S, Mestre P, Baulcombe D: An enhanced transient expression system in plants based on suppression of gene silencing by the p19 protein of tomato bushy stunt virus. Plant J 2003, 33(5):949-956.

doi:10.1186/1471-2229-14-90

Cite this article as: Dilokpimol et al:: Galactosyltransferases from Arabidopsis thaliana in the biosynthesis of type II arabinogalactan: molecular interaction enhances enzyme activity. BMC Plant Biology 2014 14:90

\section{Submit your next manuscript to BioMed Central and take full advantage of:}

- Convenient online submission

- Thorough peer review

- No space constraints or color figure charges

- Immediate publication on acceptance

- Inclusion in PubMed, CAS, Scopus and Google Scholar

- Research which is freely available for redistribution

Submit your manuscript at www.biomedcentral.com/submit
C Biomed Central 